\title{
Effect of Multiple Fingers on Receiver Performance in CDMA Forward Link
}

\author{
Twinkle V. Doshi \\ EC Dept., \\ BITS EDU Campus \\ Vernama, Gujarat, India
}

\author{
Upena Dalal, Ph.D. \\ Electronics Dept., \\ SVNIT \\ Surat, Gujarat, India
}

\begin{abstract}
Rake receiver can be implemented in any communication system, which has possibility of multipath components. Rake receiver can take advantage from these multipath components and combined all multipath components to achieve optimum result. The bit error rate (BER) performance of the Code Division Multiple Access (CDMA) cellular system based on IS-95 standard in the presence of an additive white Gaussian noise (AWGN) and interference has been investigated in this paper. The performance of the RAKE receiver is analysed considering of different number of fingers. The performance is evaluated under noise and interference for the CDMA forward link. Rake receiver is used in CDMA-based (Code Division Multiple Access) systems and can combine multipath components, which are time-delayed versions of the original signal transmission. Combining is done in order to improve the signal to noise ratio at the receiver and so reduced the BER. The BER has been studied for different numbers of Rake fingers.
\end{abstract}

\section{Keywords}

BER, Rake Receiver, Multipath, CDMA, IS-95, fingers

\section{INTRODUCTION}

CDMA (Code Division Multiple Access) spread spectrum systems, is such that, the chip rate is typically much greater than the flat fading bandwidth of the channel. Whereas conventional modulation techniques require an equalizer to undo the Inter Symbol Interference (ISI) between adjacent symbols, CDMA spreading codes are designed to provide very low correlation between successive chips. Thus, propagation delay spread in the radio channel merely provides multiple versions of the transmitted signal at the receiver. If these multipath components are delayed in time by more than one chip duration, they appear like uncorrelated noise at a CDMA receiver, and equalization is not required[1].

\section{BASIC CONCEPTS}

There are two well-known modulation methods for CDMA are: SS-CDMA and FHSS. CDMA systems commonly use the spread spectrum technique with spreading codes designed to provide very low correlation between successive chips. Due to the signal propagation characteristics of the wireless communications channel, the receiver may receive one direct line-of-sight (LOS) wave and many multiple versions of the transmitted signal at a spread of arrival times. If these multipath signals are delayed in time by more than one chip interval, the dispreading process will make the uncorrelated noise appear as negligible at the receiver. This leads to the implementation of a RAKE receiver [3] within a CDMA system, as it is able to recover each multipath signal and combine them with the correct delays to achieve a significant improvement in the SNR of the output signal. The RAKE receiver however, works only on the basis that these multipath components are practically uncorrelated from one another when their relative propagation delays exceed a chip period.

IS-95 CDMA was a telecommunications standard published by the ITU in 1995 [1]. To allow subscribers of the AMPS to switch to CDMA systems, CDMA systems operates on the same frequency band as the AMPS.

Therefore, mobiles base stations can easily operate in either mode without any modification to their design [9]. In IS-95 CDMA systems, there are basically two directions of data transmission involved; the forward (from base station to mobile) and reverse (from mobile to base station) links [4]. IS-95 specified the reverse link operation in the $824 \sim 849$ $\mathrm{MHz}$ band and $869 \sim 894 \mathrm{MHz}$ for the forward link. Each forward and reverse link channel pair is separated by $45 \mathrm{MHz}$ and the maximum user data rate is $9600 \mathrm{bps}$ [4].

\section{FORWARD LINK OF IS-95 SYSTEM}

The Forward Link transmitter inter operations comprise of convolution encoding and repetition, block interleaving, long PN sequence, data scrambling, Walsh coding and quadrature modulation [1].

Table 1. Forward Link Channel Parameters[7]

\begin{tabular}{|l|c|c|c|c|}
\hline \multicolumn{1}{|c|}{ Parameter } & \multicolumn{4}{|c|}{ Data Rate(bps) } \\
\hline User data rate & 9600 & 4800 & 2400 & 1200 \\
\hline Coding Rate & $1 / 2$ & $1 / 2$ & $1 / 2$ & $1 / 2$ \\
\hline $\begin{array}{l}\text { User Data } \\
\text { Repetition Period }\end{array}$ & 1 & 2 & 4 & 8 \\
\hline $\begin{array}{l}\text { Baseband coded } \\
\text { Data Rate }\end{array}$ & 19,200 & 19,200 & 19,200 & 19,200 \\
\hline $\begin{array}{l}\text { PN Chips/Coded } \\
\text { Data Bit }\end{array}$ & 64 & 64 & 64 & 64 \\
\hline PN Chip Rate & 1,2288 & 1,2288 & 1,2288 & 1,2288 \\
\hline PN Chips/Bit & 128 & 256 & 512 & 1024 \\
\hline
\end{tabular}




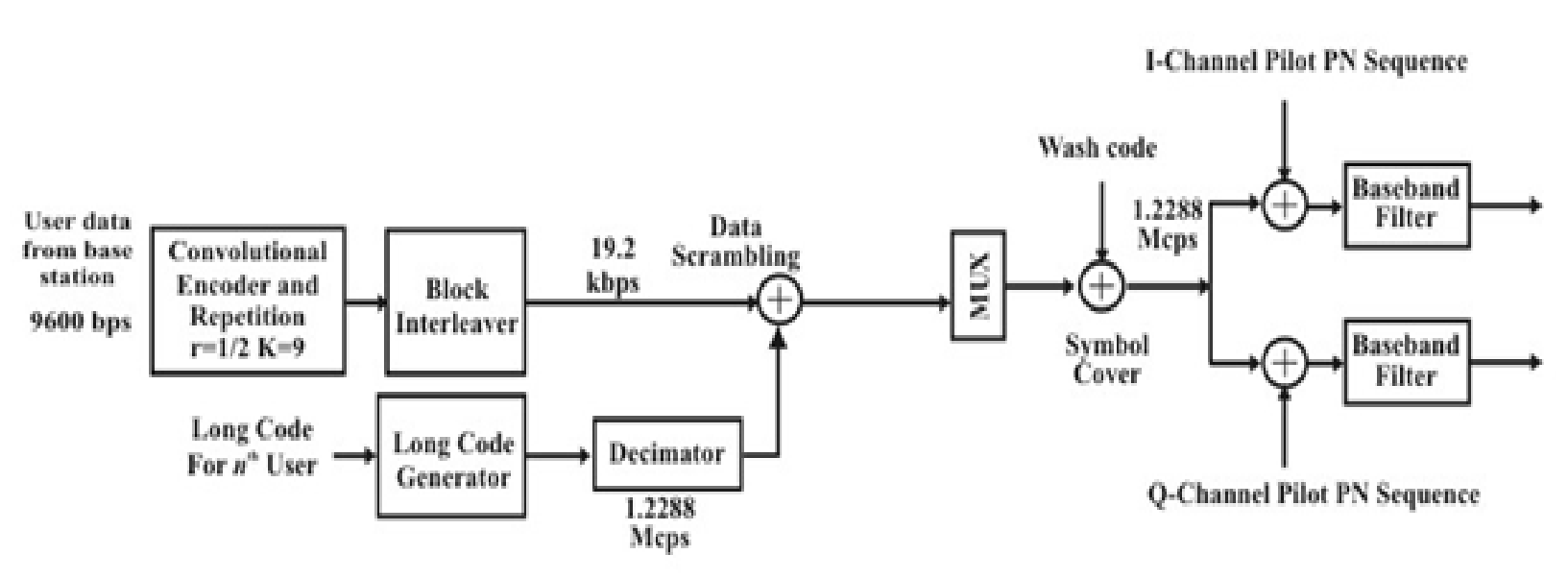

Figure-1 IS-95 CDMA forward link transmitter modulation process. [1]

The Convolution encoder performs error-control coding by encoding the incoming bit stream information. This allows error correction at the receiver, and hence improves communication reliability [1].

After convolution encoding and repetition, symbols are sent to a $20 \mathrm{~ms}$ block interleaver which is a 24 by 16 array matrix [1]. The block interleaver rearranges the order of the bits in the transmitting data bit stream. The 384 data bits contained in each $20 \mathrm{~ms}$ data frame is consecutively read into the block interleaver row-by-row. Then, as the block interleaver matrix is totally filled up, the block interleaver outputs the data bits read column-by-column.

As a result, block interleaving greatly decreases the transmitting user information's susceptibility to error bursts, and hence increases the probability of recovering the original user message at the receiver [1].

A Long PN sequence is uniquely assigned to each user and it is a periodic long code with period $242-1$ [4]. There are two reasons for using the long $\mathrm{PN}$ sequence.

1. Channelization: The base station separates forward channel traffic by applying different sequences to different subscribers.

2. Privacy : Each user uses different long codes, and due to the pseudorandom nature of the codes, hence they are difficult to decode as different sequences are orthogonal to each other.

Data scrambling is performed after the block interleaver [1]. The $1.2288 \mathrm{cps}$ long PN sequence is applied to a decimator, which keeps only the first chip out of every sixty-four consecutive PN chips. The symbol rate from the decimator is $19.2 \mathrm{ksps}$. Data scrambling is performed by modulo- 2 addition of the interleaver output with the decimator output symbol [1].

Walsh coding is performed after data scrambling in the transmitter. Each data symbol coming out of the scrambler is replaced by 64 Walsh chip sequence.

The final processing before transmitting the user's information involves Quadrature modulation of the Walsh coded data chip stream [1]. Quadrature modulation allows easy acquisition and synchronization at the mobile receiver [1].

Quadrature modulation involves separating the incoming data chip stream into an I data chip stream and a Q data chip stream and mixing each with their corresponding short PN sequences.

\subsection{Transmitter of Forward Link}

The Forward Link transmitter inter operations comprise of convolution encoding and repetition, block interleaving, long PN sequence, data scrambling, Walsh coding and quadrature modulation [1]. A diagram of the transmitter modulation process is shown in "Figure 1".

\subsection{Fading in Communication Channel}

The term "fading" describes the small-scale variation of a mobile radio signal. As each transmitting signal is represented by a number of multipaths and each having different propagation delays, the channel impulse response is different for each multipath. Therefore, not only the channel response is time varying, the channel response is also functional dependent on the propagation delay. Hence, the channel impulse response should actually be $t$ and $t$ summarised as $h(t, \tau)$, which $t$ is the specific time instance, and $\tau$ is the multipath delay for a fixed value of $t$. As a result, the received signal in a multipath channel consists of a number of attenuated, time delayed, and phase shifted versions of the original signal, and the baseband impulse response of a multipath channel can be written as [5]:

$h_{b}(t, \tau)=\sum_{t=0}^{N-1} a_{i}(t, \tau) \exp \left[j\left(2 \pi f_{c} \tau_{i}(t)+\phi_{i}(t, \tau)\right)\right] \delta\left(\tau-\tau_{i}(t)\right)$

Where ai $(\mathrm{t}, \tau)$ and $\tau \mathrm{i}(\mathrm{t})$ are the amplitude and delay, respectively, of the $i$ th multipath component at time t. The phase term $2 \pi f_{c} \tau_{i}(t)+\phi_{i}(t, \tau)$ represents the phase shift due to free space propagation of the ith multipath component, plus any additional phase shift which it encountered in the channel. And $\delta\left(\tau-\tau_{i}(t)\right)$ is the unit impulse function for the ith multipath ith component with delay $\tau$ and at time instance t.

\subsection{AWGN Noise}

Noise is often classified as some wanted signals or interference, which is present along with an information signal in a communication channel. And often, the level of noise present is incontrollable, as there are so many potential sources of noise in the channel However, by determining the approximate power level of noise in a communication channel, the Bit-Error-Rate (BER) of a communication system can be greatly reduced, by 
adjusting the power level of the transmitting information signal. In IS-95 CDMA cellular system, the channel noise analysis is often based on white noise.

White noise is a type of noise that is often exists in communication channels. It is remarkably different from any other types of noise, due to the fact that it's Power Spectral Density (PSD) is independent of the operating frequency.

In addition, if the white noise is also Gaussian, then it's also statistically independent, and exhibit total randomness. In common IS-95 CDMA forward link cellular systems, communication channels are often modeled with Additive W hite Gaussian Noise (AWGN).

\subsection{Receiver of Forward Channel}

The IS-95 CDMA standard describes the processing performed in the terminal receiver as being complementary to those of the base station modulation processes on the Forward CDM A Channel [4]. The demodulation processing that the terminal receiver architecture must perform includes Rake receiver combining (IQ demodulation and maximal combining), Walsh decoding, long PN sequence, data descrambling, block-deinterleaving, and Viterbidecoding. These operations all act to reverse the operation of one of the corresponding components in the transmitter as shown in "fig. 2 ".

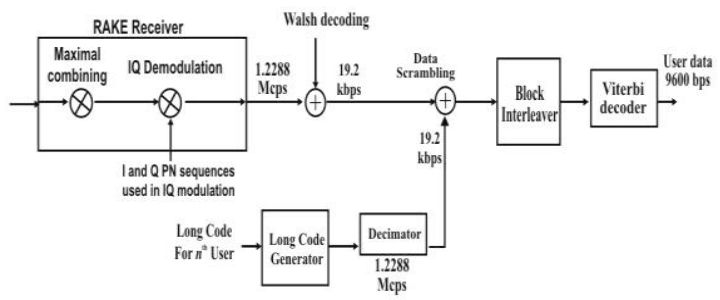

Figure 2 - IS-95 CDMA forward link receiver demodulation process. [1]

There are three major operations carried out in the RAKE receiver: (1) capturing the delayed versions of the receiver signals, (2) IQ demodulation using the reference short PN sequences which are used in IQ-modulation in the transmitter and (3) assigning weights to the correlator outputs and performing maximal combining to retrieve a final signal

1. Each correlator receives a different delayed version of the received signal. Each correlator picks up a different delayed version of the user information signal.

2. In order to pass the received signal from the input to the output of a correlator, the correlator must apply the reference pilot $\mathrm{PN}$ sequences used in the IQ modulation stagein the transmitter. In fact, the operation of IQ demodulation is the same as performed in IQ modulation, by simply applying the same I and Q pilot PN sequences to the received data.

3. The correlator outputs are weighted according their signal strengths. The strong paths are emphasized while the paths with no substantial contribution are suppressed. Then, all correlator's output are combined using the maximal ratio combining principle:

The signal-to-noise ratio of a weighted sum is maximized when the amplitude weighting is assigned in proportion to the pertinent signal strength, where each element of the sum consists of a signal plus additive noise of fixed power [5].

"Fig. 3" shows the model of a RAKE receiver with three correlators. This RAKE receiver design is used in the IS-95 system, where each of the three strongest time-shifted multipath signals is demodulated and weighted independently. The spreading code in the despreading process needs to be synchronized to the delay spread of the multipath signal, so that the outputs of each correlator can be summed to produce a stronger and more accurate signal. Note that in a RAKE receiver, if the outputs from one correlator are corrupted by fading, the corrupted signal may be discounted through the weighting process. Decisions based on the combination of the three separate correlator outputs are able to provide a form of diversity, which can overcome fading and thereby improve the CDMA reception[9].

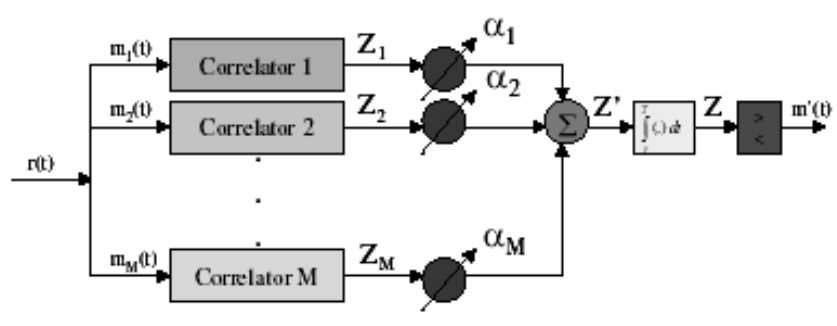

Figure3. An M-branch RAKE Receiver Implementation [1]

The $\mathrm{M}$ decision statistics are weighted to form an overall decision statistic as shown in Figure 2. The outputs of the M correlators are denoted as Z1, Z2,.., and ZM. They are weighted by $\alpha 1, \alpha 2, \ldots$, and $\alpha \mathrm{M}$, respectively. The weighting Coefficients are based on the power or the SNR (Signal-toNoise Ratio) from each correlator output. If the power or SNR is small out of a particular correlator, it will be assigned a small weighting factor, $\alpha$. If maximal-ratio combining is used, following equation 2 can be written for Z' [8].

$$
Z^{\prime}=\sum_{m=1}^{3} \alpha_{m} Z_{m}
$$

\subsection{Processing at Receiver side}

Viterbi decoding is the final processing to be performed at the receiver, and in the entire communication system. The purpose of the Viterbi decoding is to decode the convolutional encoded user data symbols. And the Viterbi algorithm used is the equivalence between maximum likelihood decoding and minimum distance decoding for a binary symmetric channel [5] [6].

In brief, maximum likelihood decoding means the decoder must choose the estimate $\mathrm{c}$ for which the log-likelihood function $\log p(r \mid c)$ is maximum, where $r$ is the receied code vector and $\mathrm{c}$ is the trnamitted vector. On the other hand, minimum distance decoding means a decoder must choose the estimate $\mathrm{c}$ that minimizes the Hamming distance between the received vector $\mathrm{r}$ and the transmitted vector $\mathrm{c}$ [5] [6].

Hence, the Viterbi algorithm implies that a convolutional code can be decoded by selecting a path in the code tree, whose coded sequence differs in the least number of places from the received sequence, or essentially, the Hamming distance between the two codes. 
The Viterbi decoder user in common IS-95 CDMA forward link transmitter has a window length of 18 . Therefore, every 18 bits of convolutional encoded user data bit stream enters the Viterbi decoder and decoded. As a result, 9 bits are produced at the decoder output after each decoding.

The algorithm works by predicting all the outputs of the convolutional encoder based on the starting shift register states of the encoder, and find the output that has the least difference when compared to the incoming 18 data bits sequence into the decoder. For every $20 \mathrm{~ms}$ user data frame, the Viterbi decoder initially computes a code tree, with the knowledge that the shift register of the convolutional encoder starts with all 0 's, then compute all 512 possible 18 bits output of the encoder after 9 iterations. Then, the decoder will compare the incoming 18 bits sequence, will all the 512 possible outputs and select the one with the least Hamming distance. Then, the Viterbi decoder regenerate its code tree by assuming the starting state of the convolutional encoder with the starting state of the 18-bit output previously selected. This process is repeated until a total of 21 decoding operations executed, which outputs a total of 378 bits. And finally, an extra of 6 zero bits are output from the decoder to give a total of 384 bits.

\section{EXPERIMENTAL RESULT AND ANALYSIS}

In the RAKE receiver selection of the number of fingers is the crucial thing. As number of finger increses the computational complexity also increses and with small number of fingers the performance is not satisfactory. In this section, the effects of increasing the finger of rake receiver has been investigated. The systems ability to be immune to multiuser interference is due to the fact that interfering signals of other users uses (1) different PN sequence, (2) different Walsh code and (3) different I and Q pilot sequences. Although these interfering signals have contribution in raising the noise background, however they are unable to affect the BER performance of the communication system, provided the transmitter transmits at an adequate power level to keep the SNR in the communication at least above the cutoff level

\section{CONCLUSION}

The design and implementation of the simulation package and the testing on the effects of increasing the number of Rake fingers have on the simulated communication system had been discussed here. The interfering signals have contribution in raising the noise background, but they do not affect the BER performance of the communication system, provided the transmitter transmits at an adequate power level to keep the SNR in the communication at least above the cutoff level, as proven by results. Also the performance is good as number of rake fingers increases. The simulation is done for the line of sight(CM1) scenario. The same can be verified for the different channel conditions. The different optimization techniques like NLMS, Honeybee algorithm can be employed to achieve better result.

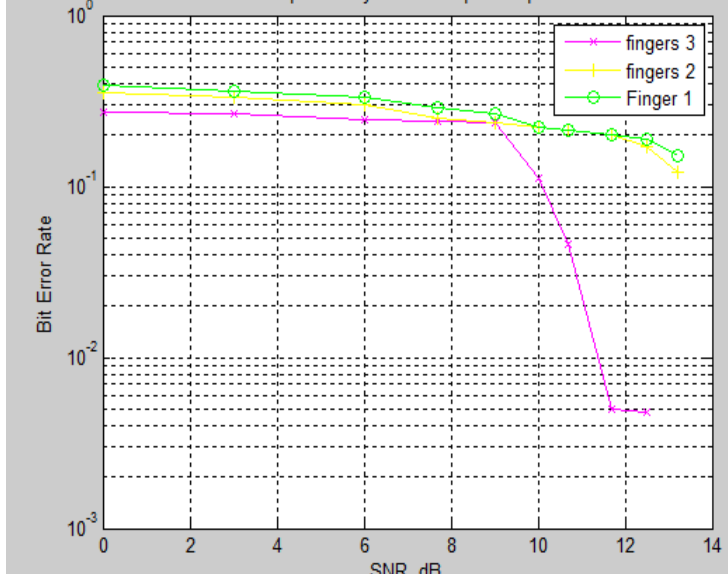

Figure4: Effect of increasing number of Rake Fingers

\section{REFERENCES}

[1] Rappaport, Wireless Communications Principles and Practice, Prentice Hall, New Jersey, 1996, pp. 336-338.

[2] Proceedings of the Design, Automation and Test in Europe Conference and Exhibition Designers' Forum (DATE'04) 1530-1591/04 \$20.00 @ 2004 IEEE

[3] Mosa Abu-Rgheff, 2007, Introduction to CDMA wireless communication.

[4] Widrow, B., \& Stearns, S.D. 1985. Adaptive Signal Processing. Prentice Hall: New Jersey.

[5] S. Haykin, Communication Systems, John W iley \& Sons, 2001

[6] A. J. Viterbi, - CDMA principles of spread spectrum communication", Addison-Wesley Publishing Company, 1995.

[7] Sanjeev Kumar, Monika Tuteja \& Ramneek Singh,” BER Performance Analysis of CDMA Reverse Link under AWGN Channel", International Journal of Computer Applications (0975 - 8887) Volume 21- No.2, May 2011

[8] Doshi, T.V.; Zalawadia, K.R.; Dalal, U.D., "BER Performance Analysis of CDMA Forward Link," Computational Intelligence and Communication Networks (CICN), 2011 International Conference on, vol., no., pp.233,237, 7-9 Oct. 2011 doi: 10.1109/CICN.2011.46

[9] Doshi, Twinkle V.; Zalawadia, Khyati R., "Performance analysis and comparison of different RAKE receivers for UWB channel," Emerging Technology Trends in Electronics, Communication and Networking (ET2ECN), 2012 1st International Conference on, vol., no., pp.1,5, 19-21 Dec. 2012 doi: 10.1109/ET2ECN.2012.6470090 